\title{
Victor-Laurent Tremblay
}

\author{
Wilfred Laurier University
}

\section{Artaud, schizophrène universel}

ans Les Nourritures terrestres, Gide conseillait à Nathanaël de jeter son livre afin qu'il puisse trouver sa propre vérité. Plus tard, un Sartre vieilli, détruisait dans Les Mots la statue de monstre sacré d'une religion littéraire qu'il avait aidé à fonder au cours de sa vie. L'humilité, sinon le rejet, de ces auteurs face à leur oeuvre, Artaud, un de leurs contemporains, l'a universalisée jusqu'à la dégradation: "Toute l'écriture est de la cochonnerie," affirme-t-il (1: 120). Une analyse des écrits d'Artaud est donc paradoxale, non seulement parce qu'elle va à l'encontre de son désir, mais parce que son oeuvre n'est ni romanesque, ni poétique, ni même théâtrale (une absence d'oeuvre selon Foucault [555]), mais bien plutôt une longue autobiographie racontant une destinee: "Ce que vous avez pris pour mes oeuvres n'était que les déchets de moi-même, ces raclures de l'âme que l'homme normal n'accueille pas" (1: 114).

Pourtant lire Artaud sous un éclairage psychanalytique est un sacrilège nécessaire. En réalité, il ne s'agit point d'expliquer son oeuvre à l'aide de quelques complexes freudiens. Artaud n'a pas besoin de la psychanalyse, c'est elle qui a besoin de lui. Il s'est en effet auto-analysé avec une telle lucidité dans sa "chose littéraire" que la psychanalyse peut y retrouver et corroborer la matière même de ses recherches. Ainsi l'expérience "vitale" de l'homme Artaud, s'interrogeant sur les mécanismes de la pensée et sur l'emprise du langage sur celle-ci, se transforme en une observation universelle de l'homme et de ses rapports avec la réalité.

"De l'homme à l'homme vrai, le chemin passe par l'homme fou", dit Foucault (544), rappelant que tous les progrès vers une meilleure connaissance de la psyché de l'homme se sont effectués grâce à l'analyse de troubles mentaux. Aussi la folie qu'Artaud oppose au "mode intégralement embourgeoisé" (9: 180) ouvre-t-elle des portes 
peut-être inquiétantes mais qu'il importe de franchir. Mettant de côté le mythe Artaud, nous nous sommes donc mis à l'écoute de ses cris et "supplications", utilisant Julia Kristeva comme guide, car il semble que la démarche littéraire de celle-ci se déroule parallèlement au projet artaldien. Le sémiotique, se constituant des formes du désir qui traversent le symbolique pour s'inscrire dans le langage ou l'écrit, n'est-ce pas ce qu'Artaud rêve d'atteindre et d'exprimer? Il essaiera en effet toute sa vie de révéler ce "double", de traduire cette "nonpensée" surgissant du plus profond de lui-même, s'opposant au mode bourgeois et intellectuel, bâti d'ailleurs sur le rejet de cet "innommable".

\section{Conscience d'une "non-pensée":}

Si plus que tout autre, Artaud s'est opposé à la psychanalyse, ce n'est pas en tant qu'instrument de découverte de soi, mais en tant que moyen de "normalisation" utilisé par la société pour rendre un individu productif, pour l'intégrer à "ce monde puant [...] de l'ineptie bourgeoise” (9: 180). De la même façon qu'il s'insurge contre les "investigations dans le domaine de l'esprit" par la psychiatrie, (1 Supplément: 186), Artaud dénonce les rapports de contrainte existant entre le langage et la société, celle-ci n'étant qu'un instrument de propagande constitué par les calques idéologiques de celui-là (les mots) qui emprisonnent la vie:

[...] la culture n'est pas écrite, et [...] comme le dit Platon: "La pensée a êté perdue du jour où une parole a été écrite." Ecrire c'est empêcher l'esprit de bouger au milieu des formes comme une vaste respiration. Puisque l'écriture fixe l'esprit et le cristallise dans une forme, et, de la forme, naît l'idolâtrie. (8: 203)

Sous cette expérience et un vocabulaire différent, à partir de la dialectique hégélienne, l'on reconnaît les antagonismes lacaniens. Pour Hégel, afin de construire un ordre objectif propre à l'historicité, il faut détruire l'unité subjective. On assiste ainsi à un mouvement de scission, de négativité affirmative, créateur d'être mais basé sur 
du néant. Cette première négativité, à la charnière de l'ordre biologique et de l'ordre social constitue, selon Lacan, le "sujet unaire", c'est-à-dire celui qui est soumis à la loi du UN, laquelle est déterminée par le manque, le rejet du désir dans l'inconscient. Cette censure consolide le moment paranoïde du sujet qui se transforme alors en sujet spéculatif, étatique et religieux, soumis ainsi à tous les systèmes patriarcaux. ${ }^{1}$

Pour Kristeva, cette première négation de la chora pulsionnelle (désir de rejoindre l'unité psychobiologique bébé-mère), lieu où le sujet est encore multiple et qui suppose une "pensée impersonnelle" originale, va produire le sémiotique, "langage" sans parole du corps originel pour lequel Artaud désespère de nostalgie: ${ }^{2}$

Vous n'imaginez pas à quel point je puis être privé d'idées. Je n'ai même pas les idées qui pourraient correspondre à ma chair, à mon état de bête physique, soumise aux choses et rejaillissant à la multiplicité de leurs contacts. (1: 348)

Le sémiotique qui se définit comme un ordonnancement vocalique et gestuel, réglé par les contraintes biologiques, sexuelles et familiales, est un espace rythmique, "musical", structurable, qui sans cesse se fait et se défait. Il se situe antérieurement à la signification, au signe, à la position du sujet dans le langage, préalable à son entrée dans l'ordre symbolique où s'effectue l'identification du sujet. Causé par la négativité première (manque de la mère, séparation d'avec la choral, il est surgissement de l'inconscient vers l'extérieur; à la fois désirable et répugnant (car un retour complet amènerait la folie, la mort), il correspond à l'abjection.

La fonction symbolique articulée par le sémiotique a cependant une autre origine. Elle naît d'une seconde négation associée à la pulsion anale vers l'âge de quinze mois. Cette interdiction de l'appropriation de la matière fècale coïncide avec l'apparition du langage. Le non sémantique, lieu de surmoi paternel, renforce le "sujet unaire" dans sa négation au plaisir qui, sublimé, pourra accéder au jeu artistique (et linguistique) grâce à la symbolisation. 
Artaud ne connait rien de ce processus de la signifiance, mais, en affirmant ce qu'il ressent au plus profond de son être, il met en procès ces instances négatives qui dissolvent l'unité subjective. Il commencera par mettre en doute le langage, gardant cependant la poésie comme lieu privilégié, car plus libérée des règles de la langue, permettant à des flots de pulsions sémiotiques de se glisser à travers ces brèches:

Tout sentiment puissant provoque en nous l'idée du vide. Et le langage clair qui empêche aussi la poésie d'apparaître dans la pensêe. C'est pourquoi une image, une allégorie, une figure qui masque ce qu'elle voudrait révéler ont plus de signification pour l'esprit que les clartés apportées par les analyses de la parole. (4: 86)

Il se rendra cependant vite compte des contraintes nécessaires exigées par l'utilisation des mots eux-mêmes:

Le poète qui écrit s'adresse au Verbe et le Verbe a ses lois. Il est dans l'inconscient du poète de croire automatiquement à ces lois. Il se croit libre et il ne l'est pas.[...] Je ne veux pas d'un verbe venu de je ne sais quelle libido astrale et qui fut toute consciente aux formations de mon désir en moi. ("Révolte contre la poésie," 9: 143-44)

Aussi Artaud rêve-t-il de l'avènement de la Chair qui se fera Verbe: espérance de retour à la chora primordiale, union à la mère, saut dans le vide:

J'imagine un système où tout l'homme participerait, l'homme avec sa chair physique et les hauteurs, la projection intellectuelle de son esprit. [...] Il y a des cris intellectuels, des cris qui proviennent de la finesse des moelles. C'est cela, moi, que j'appelle la Chair. Je ne sépare 
pas ma pensée da ma vie. Je refais à chacune des vibrations de ma langue tous les chemins de la pensée dans ma chair. (1: 351-52)

C'est si l'on veut la connaissance par le vide, une espèce de cri abaissé et qui au lieu qu'il monte descend. Mon esprit s'est ouvert par le ventre, et c'est par le bas qu'il entasse une sombre et intraduisible science, pleine de marées souterraines, d'édifices concaves, d'une agitation congelée. (1: 350)

\section{Un conflit douleureux:}

Cet affreux combat intérieur entre l'UN social (patriarcal), législateur de langage, et la chora primitive (maternelle) coûtera à Artaud de nombreuses années d'internement, mais plus encore, de douleurs lucides à plusieurs niveaux, et cela, depuis sa jeunesse, souffrances formulées pour la première fois dans sa Correspondance avec Jacques Rivière (1923) et décrites, minutieusement et avec rage, dans ses premières oeuvres: L'Ombilic des Limbes (1925), Le Pèse-Nerfs (1925), Bilboquet (différentes époques) qu'il résumera, en 1932, en ces termes:

Je souffre [...] / 1\$ intellectuellement, d'une part, / 2\$ sentimentalement et affectivement, de l'autre. / [...] Si je pense, la majeure partie des réserves de termes et du vocabulaire que je me suis personnellement constitué est inutilisable, étant rouillée et oubliée quelque part, mais le terme apparu, la pensée profonde cède, le contact est brutalement coupé, l'affectivité nerveuse profonde ne répond plus à la pensée, l'automatisme est désorganisé, et cela est pour les fois où je pense !!! [...] drame intellectuel où je suis perpétuellement vaincu $[. .$.$] si intellec-$ tuellement mon cerveau est devenu inopérant, ne peut plus servir, les moments pendant 
lesquels ce vide m'occupe me remplit d'angoisse et d'ennui, me fait sentir ma vie perdue, inutilisable, ont une valeur sentimentale eux aussi, ils se traduisent pour l'âme par une coloration de néant. ${ }^{3}$ [.] (1 Supplément: 131-33)

A ce déssaroi intellectuel et affectif, le corps participe. Ses faiblesses mentales "ont des racines vivantes, des racines d'angoisse qui touchent au coeur de la vie" (1: 52 ). La maladie non seulement "déracine la pensée" mais lui enlève la parole et désorganise le corporel, l'obligeant à se droguer pour atténuer la souffrance:

Il y a un mal contre lequel l'opium est souverain et ce mal s'appelle l'Angoisse, dans sa forme mentale, médicale, physiologique, logique ou pharmaceutique, comme vous voudrez. / L'Angoisse que fait les fous. / L'Angoisse qui fait les suicidés. / [...] L'Angoisse que la médecine ne connait pas. / [...] L'Angoisse qui pince la corde ombilicale de la vie. [...] ("Lettre à Monsieur le Législateur de la loi sur les stupéfiants", 1: 83)

Déchirement, donc, inscrit douloureusement dans son corps, entre deux cyclones qui veulent l'avaler: d'un côté, le dégoût de tous les contrôles sociaux le sollicitant, incluant le langage dont il a pourtant un besoin vital pour retenir et formuler sa pensée, et de l'autre, ce gouffre de la chair multiple qui l'appelle incessamment, qu'il vénère et désire, mais qui, dangereuse et répugnante, peut l'engloutir.

L'approche de cet objet, objet du refoulement originaire, le fait errer dans les territoires réservés à l'animal, immergés dans la sexualité et la violence, l'érotisme et la douleur. "Sujet unaire" s'il acceptait les systèmes dont le bourgeois bien-pensant est le parfait prototype, Artaud sans choisir devient "sujet en procès", il les remet en question. Cette dialectique entre la tentation du corpores et les contraintes du rationnel se traduit avec force et douleur dans son oeuvre et s'amplifie inexorablement avec le temps. Ainsi il dira en 1947 de ses cahiers écrits à Rodez, lors des trois années de son 
internement, qu'ils "sont pleins d'exclamations, d'interjections, d'abois, de cris, sur l'antinomie entre vivre et être, agir et penser, matière et âme, corps et esprit" $(9: 174)$.

Impuissant, déchiré, Artaud pour quelque temps cherchera à assouvir sa soif d'absolu dans une femme, Génica Athanasiou, de descendance grecque comme sa mère. Refusant d'abord de se courber sous la duplicité, même fascinante, de la sexualité, il perçoit enfin dans le domaine de la chair, un lieu de rencontre qui s'appuierait sur les possibilités du sexe pour transformer le corps en extase à travers l'obscénité (attirance de l'abjection, objet du désir refoulé, la mère). Mais il se rend compte que les sommets sensuels sont rarement atteints. Le ciel érotique alors se résorbe sur lui-même en nuit fourmillante avec son cortège d'égoûts: "[...] quelle belle image qu'un châtrée" écrit-il en s'identifiant à Abélard (1: 137). L'amour n'a pas su garder ses promesses:

Toutes nos sensations seront-elles à jamais intellectuelles, et nos rêves n'arriveront-ils pas à prendre feu sur une âme dont l'émotion nous aidera à mourir. Qu'est-ce que cette mort où nous sommes à jamais seuls, où l'amour ne nous montre pas le chemin? (1: 152)

La solution pourtant n'est pas le suicide qui n'est possible, selon lui, que dans la lucidité totale d'un homme qui sait tout des choses. N'étant que doute et ignorance, "l'état proprement dit du suicide est pour [lui] incompréhensible." Son cri, "ON m'a [déjà] suicidé" (1:318), est un rappel du refoulement primaire chez l'enfant, et c'est avec nostalgie qu'il contemple ce paradis perdu:

Mais que penseriez-vous d'un suicide antérieur, d'un suicide qui nous ferait rebrousser chemin, mais de l'autre côté de l'existence, et non pas du côté de la mort. Celui-là seul aurait pour moi une valeur. Je ne sens pas l'appétit de la mort, je sens l'appétit du ne pas être, de n'être jamais tombé dans ce déduit d'imbécilités, d'abdications, de renonciations et d'obtuses 
rencontres qui est le moi d'Antonin Artaud bien plus faible que lui. (1:318)

Prophétisant son "suicide antérieur" futur dans la folie, Artaud, cependant, qui présume de ses forces, va tenter de se débarrasser de "ce moi béquillard", ce moi social sur l'ombre duquel il crache, mais "qui se retrouve tout de même dans la réalité" (1: 318$)$.

\section{Révolte contre la réalité:}

Pendant un certain temps, Artaud s'attarde donc à la tâche de refaire le monde en joignant le groupe des surréalistes. Al'homme "mal dans son corps" fait alors place un révolutionnaire, un terroriste de l'esprit. Sous forme de tract, il prend l'initiative d'une déclaration collective:

$1^{\circ}$ Nous n'avons rien à voir avec la littérature; / Mais nous sommes très capables, au besoin, de nous en servir comme tout le monde.

$2^{\circ} \quad$ Le surréalisme [...] est un moyen de libération totale de l'esprit / et de tout ce qui lui ressemble.

$3^{\circ}$ Nous sommes bien décidés à faire une Révolution. [...]

$5^{\circ}$ Nous ne prétendons rien changer aux moeurs des hommes, mais nous pensons bien leur démontrer la fragilité de leurs pensées, et sur quelles assises mouvantes, sur quelles caves, ils ont fixé leurs tremblantes maisons. [...]

$8^{\circ}$ Nous sommes des spécialistes de la Révolte. [...] (1: 325-26)

Cet appel aux armes, contre la pensée logique conventionnelle, montre bien qu'Artaud est dégoûté de l'édifice lézardé établi par Platon-Aristote et consolidé par Aquin-Descartes. La philosophie de l'Occident, qui déteint sur nos idées, s'est fait, écrit-il en 1932, une "bien faible et bien mesquine idée de l'être, pour avoir osé appeler moi cette partie [...] excrémentielle de l'âme où se déverse la mémoire de nos plus brutales, $[\ldots]$ de nos plus instinctives émotions" (8: 17).

Lorsque lui est confiée la direction du troisième numéro de la Revolution surréaliste, il écrit des textes d'une grande virulence, sans pourtant les signer. Sous le titre provocateur de la couverture, 1925: Fin de l'ère chrétienne, il accuse les coupables auxquels il adresse 
des lettres: le Pape, le Dalaï Lama, le Bouddha et les recteurs des universités européennes, tous maîtres de la pensée. ${ }^{4}$ Le surgissement de cette violence dans le texte est l'autre pendant du "procès de la signifiance". Après l'angoisse mortelle ressentie, par enflement paranoïde, le moi s'affirme dans une "unité productrice": "un effort perpétuel / de domination / d'exaltation / d'abolition / de précision / d'appétit / de désir / informulés / de transformation" (cité dans Kristeva, "Le Sujet" 82-25).

Habituellement, cette phase d'affirmation chez l'individu renforce les rejets primaire et secondaire par l'acceptation totale de la loi de l'UN. Bien que ce processus de négation implique le rejet sexuel du parent du même sexe, sous la contrainte biologique et sociale, l'enfant a tendance à s'identifier à celui-ci, créant ainsi une situation de va-et-vient propre à la symbolisation et à la signifiance, parallèle au rôle de la mobilité entre l'attrait vers le sémiotique et son rejet. (Kristeva rappelle en effet que l'homosexualité refoulée se cache non vue sous l'UN, à la base de la normativité et de la normalité sociale ["Le Sujet" 84].)

Tout sujet en procès, comme Artaud, "transporte, sans la sublimer, la charge de rejet dans le mouvement même qui le fait traverser les interdits et les institutions sociales: dans le mouvement d'une pratique révolutionnaire (politique, scientifique ou artistique)". (Kristeva, "Le Sujet" 85). L'animosité qu'Artaud montre contre la "prêtraille" et contre le "Ip]ère, patrie, patron", base quadripartie de la "vieille société patriarcale et [de] la chiennerie fasciste" s'explique par son refus de la négativité, du refoulement (8: 172; voir aussi 12: 96). L'agressivité, si longtemps repoussée ou sublimée, causée par le manque primordial de la mère, peut alors librement s'exprimer contre toute figure autoritaire qui perpétue la pédérastie refoulée du père au fils, inscrite dans la Loi. Bien que, s'appuyant encore sur le système linguistique pour transmettre sa fureur, Artaud, qui reconnait le pouvoir de contrainte de celui-ci par la symbolisation, s'en méfie:

[J]'aime les poèmes jaillis et non les langages cherchés. Je veux, quand j'écris ou que je lis, sentir bander mon âme [...]. Je n'aime pas les poèmes ou les langages de surface et qui 
respirent d'heureux loisirs et des réussites de l'intellect [...]. (9: 184)

Kristeva, en sémanalyse, qualifie de phéno-texte la structure plate (ou de surface du texte) qui permet le passage d'un message, le récit d'une histoire quelconque (217-18). Au contraire, le géno-texte est constitué de structures moins visibles, agencées par la chora sémiotique. Artaud en détruisant le phéno-texte, qui porte l'idéologie et la logique, permet au géno-texte d'accéder à la conscience. Ainsi son agressivité échappe-t-elle à la fonction symbolique pour s'inscrire dans le signifié, de même que dans le signifiant, par des phrases hachées et rapides, à caractère oral où s'active une jouissance de destruction dont voici un exemple:

Le Confessionnal, ce n'est pas toi, ô Pape, c'est nous, mais, comprends-nous [...] Nous n'avons que faire de tes canons, index, péché, confessionnal, prêtraille, nous pensons à une autre guerre, guerre à toi, Pape, chien. (1:338)

Depuis longtemps Artaud s'est voué à la chasse aux préjugés, ces "vices de nature et vices de forme, ces toxines de notre activité mentale, [...] mais la complétude viendra du redressement des torts sociaux et moraux de leurs prolongements" (1: 242-43). C'est pourquoi il refuse que le mouvement surréaliste s'insère dans un contexte temporal et social, surtout sous domination marxiste. Selon lui, l'activité révolutionnaire devrait aider à la naissance du rêve et de l'inconscient dans la culture et non de rendre-l'homme "prisonnier dans le cadre de ses plus grossières nécessitês” (8: 179). Il faut donc percevoir sa démission du groupe surréaliste, non comme un signe d'apolitisme et de désintérêt pour l'humanité en misère ( $c f$. "La Faim n'attend pas..." [8: 11-151]), mais comme une affirmation de liberté totale, révolte contre toute emprise surtout idéologique, car rationnelle et patriarcale. ${ }^{5}$

Evidemment, en dehors de tout système sécurisant, l'homme demeure à jamais disponible à la douleur, mais il a l'avantage de garder sa conscience et sa sensibilité. Pour demeurer en contact avec la vie, il faut accepter la déchirure "schizophrénique" (du grec 
skhizein "fendre" et phren "esprit") inscrite en l'homme entre son corps et sa "raison" dans l'espoir d'une unification ou plutôt d'une mobilité constante entre les deux.

Lorsqu'il récrit Le Moine de Lewis, par besoin d'argent, c'est que ce travail lui permet, une fois de plus, de bousculer la "réalité" à pleins bras. Dans le même mouvement de libération contre les forces idéologiques et sociales de refoulement, il accentue, autant qu'il est possible, la violence et l'atrocité du récit:

[...] je ne me souviens dans aucune lecture avoir vu [...] s'ouvrir en moi des images avec ces sortes de plongées dans tous les dessous intellectuels de l'être, des images qui, dans leur aspect d'images traînent après elles un véritable courant de vie prometteur comme dans les rêves, de nouvelles existences et d'actions à l'infini. (6: 16)

Ce besoin de rêve et de liberté, qui l'a probablement guidé vers le cinéma et le théâtre, marquera son destin: journalière intensifiée par la drogue qui l'amènera ultimement à la folie. Il va donc se consacrer à donner une forme à cet imaginaire qui le hante, à activer et à réaliser, hors de lui-même, ce "double" intérieur.

\section{Réaliser le "double" sémiotique:}

Contre la douleur "de ce crucifiement où l'âme n'en finit plus de se perdre" (1: 142) Artaud cherche désespérément un remède. C'est au cinéma, au début encore muet, qu'il trouvera sinon un certain apaisement, du moins une façon d'incarner son "désir". Au premier abord, le cinéma lui semble être le "révélateur" idéal du réel parce qu'il implique "un renversement complet de l'optique, de la perspectíve, de la logique. [...] Il agit sur la matière grise du cerveau directement" (3: 79-80), permettant ainsi d'atteindre la conscience à travers l'oeil afin d'agir sur le corps et ses pulsions, histoire de mettre le spectateur en cause, en danger même, rejoignant l'absolu entre l'illusion et la réalité. 
Si par la suite Artaud s'accommode du cinéma sonore, c'est qu'il veut supprimer le despotisme de l'écriture vocale et mettre en oeuvre sa polyphonie, utilisant ses fléchissements pour se porter à l'écoute de sa respiration. Malheureusement le travail de la caméra va s'avérer stérile, capable seulement d'enregistrer un univers "mort, illusoire et tronçonné" (3: 104):

le cinéma à son tour [...] nous assassine de reflets; [...] filtré par la machine, [il] ne peut plus joindre notre sensibilité, nous maintient [...] dans un engourdissement inefficace, où paraissent sombrer toutes nos facultés. (Cité dans Prieur, 109)

Malgré sa déception dans le domaine d'un art sur lequel il portait de grandes espérances, Artaud joue d'une manière très expressionniste des "rôles de fous, d'individus morbides pervertis et torturés qu'il interprète avec une sorte de fougue hallucinatoire" (Morel, 125). Voulant dévorer la psychologie par les actes, le cinéma lui offre l'opportunité d'actualiser sa psyché.

L'acteur, pour lui, "est un athlète du coeur" et la sphère affective doit lui appartenir organiquement $(4:$ 154). Il "trouve dans son instinct de quoi capter et faire rayonner certaines forces [...] qui ont leur trajet matériel d'organes et dans les organes" (4: 156):

Pour se servir de son affectivité comme le lutteur utilise sa musculature, il faut voir l'être humain comme un Double [...], comme un spectre perpétuel où rayonnent les forces de l'affectivité. [...] ce double a le souvenir long. [...] c'est avec son coeur que l'acteur pense [...] Savoir qu'il y a pour l'âme une issue corporelle, permet [...] d'en retrouver l'être, par des sortes de mathématiques analogies. Connaitre le secret du temps des passions, de cette espèce de tempo musical qui en réglemente le battement harmonique [... et le retrouver par analogie grâce à] la connaissance des souffles [quel provoquer[a] 
l'âme, en faciliter|a] l'épanouissement. (4: 15658)

Dans les conseils d'Artaud en vue de préparer l'acteur à son métier, la régression psychique est facilement reconnaissable. Ce "double passionné" qu'il veut faire apparaître est à la source même de son désir: rejoindre l'unité initiale de la chora, l'interdit maternel où la différenciation sexuelle est abolie (car à un stade préverbal et préoedipien). L'acteur, comme l'analysé, redevient androgyne, mâle et femelle:

Le temps de penser à vouloir et nous projetons avec force un temps mâle, suivi sans solution de continuité trop sensible d'un temps féminin prolongé. Le temps de penser à ne pas vouloir ou même de ne pas penser et voici qu'un souffle féminin fatigué nous fait aspirer une touffeur de cave, la moite haleine d'une forêt. (4: 160)

Comme la médecine chinoise, il faut procéder "par vide et par plein. Convexe et concave. Tendu relâché. Yin et Yang. Masculin fẻminin" (4: 162).

Cette tentative d'hermaphrodisme, Artaud l'a présentée magistralement dans Héliogabale (1933). Là, à l'écoute de la force choratique dans le va-et-vient des deux sexes, il planifie un programme de révolte absolue, qui tout en prophétisant sa fin éclaire notre destinée:

[...] sous l'éloquence et sous les reconstitutions [d'Héliogabale] ma vraie nature apparaît tout de même par m'y rejoindre dans le détail de maints et maints passages, et dans la conception de la figure centrale où je me suis moi-même décrit. (7: 187-88)

Si la division en trois parties de son "Anarchiste couronné" attire notre intérêt, c'est qu'elle semble recouvrir l'aventure artaldienne entière. La dẻmoralisation systẻmatique et joyeuse de la conscience 
latine à laquelle se consacra Héliogabale n'est qu'une occasion allégorique pour Artaud de subvertir l'ordre patriarcal.

La première partie, intitulèe "Le Berceau de sperme", nous situe dans le petit royaume d'Emèse d'où vient Héliogabale. Là, "LE PRIMO GENITEUR" est la mère: "la mère est père [...] et le féminin [...] engendre le masculin" (7: 20). Cette inversion sociale et biologique, née de l'anarchie, du meurtre et de la débauche, est inscrite dans le nom même des dieux d'où provient la terminologie d'Héliogabale: hélio/soleil, principe masculin, et Elagabalus, "peut-être [...] le Désir dans la vieille cosmogonie phénicienne [résultant...] du mélange lent et multiplié des principes qui rayonnaient au fond du Souffle du Chaos," vénéré sous l'image d'un immense phallus noir (la Pierre de Lune), (7: 22; voir aussi 95-101). La métaphore du soleil noir phallique voile avec transparence la "Mère primitive", cette chora sémiotique vers laquelle Hêliogabale va tendre énergiquement non dans le Verbe Viril, mais dans le geste anarchique. Par la destruction systématique, le sang, la cruauté, la guerre, il veut mélanger et réduire les choses à l'unité primordiale (7:51).

La deuxième partie, "La Guerre des principes", explique l'origine des excès et des folies de la religion du soleil noir. Elle est réactivation dans la réalité même d'une séparation primitive d'essences que la psychanalyse situe à l'époque pré-verbale choratique. Dans cette bataille entre masculin et féminin, qu'Artaud nomme Schisme d'Irshu, "les partisans du Mâle ne croient pas en la coexistence des principes, et pour eux le Mâle intelligible [le langage] demeure seul, à l'origine de tout" (7: 140). Voilà la raison de la guerre qu'Artaud a fait sienne. "L'Anarchie", la dernière partie, décrit longuement ce double combat d'Héliogabale:

$1^{\circ}$ De l'UN qui se divise en restant UN. De l'homme qui devient femme et reste l'homme à perpétuité.

$2^{\circ}$ Du Roi Solaire dont l'homme qui accepte mal d'être moi humain. Qui crache sur l'homme et finit par le jeter à l'égout. (7: 103)

L'intense circulation de sang et d'excréments de la mort d'Héliogabale, assassiné dans les latrines, illustre bien que le retour au sein maternel ne peut s'accomplir que dans l'obscénité, la violence et la mort, et augure la mort d'Artaud elle-même, "Suicidé de la 
société" (comme ce livre qu'il publiera plus tard sur Van Gogh), atteint d'un cancer à l'anus. ${ }^{6}$ Cette "identité des contraires" réalisée non sans mal par Héliogabale, dans "une lutte obstinée et abstraite entre le Masculin et le Féminin" (7: 74) dont Artaud admirait la théâtralité, ce dernier va s'en inspirer dans son propre théâtre.

Il entreprend de développer les techniques appropriées afin de "forcer" cette rencontre entre le corps maternel et l'esprit patriarcal. Mais avant d'être violente, l'expérience théâtrale doit comporter une matérialité. Le théâtre balinais lui avait fait découvrir, en 1931, "ce côté révélateur de la matière qui semble tout à coup s'éparpiller en signes pour nous apprendre l'identité métaphysique du concret et de l'abstrait et nous l'apprendre en des gestes faits pour durer" (4: 72). Ce théâtre, qui élimine l'auteur et sa parole idolâtrée au profit du metteur en scène devenu "une sorte d'ordonnateur magique, un maître de cérémonies sacrées", ritualise "un exorcisme pour faire AFFLUER nos démons [..."] On sent dans le théâtre Balinais un état d'avant le langage et qui peut choisir son langage: musique, gestes, mouvements, mots" (4: 72-74).

Il s'agit de faire surgir le double sémiotique et de le mettre en scène (qui s'étend d'ailleurs à la salle entière), "de créer une métaphysique de la parole, du geste, de l'expression, en vue de l'arracher à son piétinement psychologique et humain. Mais tout ceci ne peut servir s'il n'y a derrière un tel effort une sorte de tension métaphysique réelle, un appel à certaines idées inhabituelles [...] qui touchent à la Création, au Devenir, au Chaos [...]" (4: 107): régression à l'Unité primordiale qui ne peut se faire cependant sans douleur, d'où le nom de "thêâtre de la cruauté":

Si le thêâtre comme les rêves est sanguinaire et inhumain, c'est [...] pour manifester et ancrer inoubliablement en nous l'idée d'un conflit perpétuel et d'un spasme où la vie est tranchée à chaque minute, où tout dans la création s'élève et s'exerce contre notre état d'êtres constitués, c'est pour perpétuer d'une manière concrète et actuelle les idées métaphysiques de quelques Fables [sur "les grands Mythes [...] noirs" 4: 38] dont l'atrocité même et l'énergie 
suffisent à démontrer l'origine et la teneur en principes essentiels. (4: 110)

Je propose d'en revenir au théâtre à cette idée élémentaire magique, reprise par la psychanalyse moderne, qui consiste pour obtenir la guérison d'un malade à lui faire prendre l'attitude extérieure de l'état auquel on voudrait le ramener. [r: 95-96]

Mimétisme, réactualisation par le théâtre à la fois du traumatisme premier de l'enfance et des éléments initiatiques que l'on peut retrouver dans les religion. "Déchirure" de la mère, souligne Artaud; parricide oedipien, ajoutera Derrida:

Les représentations des Mystères orphiques montraient des formes qui s'enflaient à conquérir les ténèbres [la chora]; ces formes avaient le visage de la nuit et prenaient l'aspect du "mal envahisseur" [1'abjection]. (8: 256)

L'origine du théâtre, telle qu'on doit la restaurer, c'est une main portée contre le détenteur abusif du Logos, contre le père, contre Dieu, d'une scène soumise au pouvoir de la parole et du texte. (Derrida, 350)

La présentation des Cenci, avec son parricide, sa "violence contre la famille, la religion et la justice, [....] protestation contre ceux qui s'en sont emparés et les ont détournés de leur but fondamental: le rapport harmonieux entre les hommes" (Brau, 159), n'était pas, selon Artaud, une vraie illustration de ce théâtre, mais elle préparait cette voie nouvelle où le texte correspond aux gestes, aux mouvements de la mise en scène, aux décors, à la musique et à la sonorisation. Cependant, sous la critique féroce, la pièce est un fiasco. Artaud, qui "ne croi[t] plus à la pureté des hommes" (5: 262) est à bout de forces et "ne sailt] plus de quel côté [se] retourner pour vivre" (5: 259). Ne 
pouvant faire jouer son thêâtre sur la scène, il ne lui reste plus, en publiant des manifestes, qu'à le défendre par l'écriture.

Le cinéma lui avait permis de se rendre compte qu'il était possible de faire sourdre ce "double" que la parole ne peut rendre accessible. Par le théâtre, il avait cru pouvoir étendre à la collectivité cette prise de conscience des seules forces vives de l'homme que la société détourne. Artaud était assuré qu'il devait son échec à la civilisation judéo-chrétienne. Son aventure mexicaine, située à la suite de ces revers, prend alors tout son sens. Peut-être chez les descendants des Mayas existait-il une culture non-matérialiste qui encourageait la libération de l'énergie de l'âme?

[...] une civilisation à bases Métaphysiques qui s'expriment dans la religion et dans les actes par une sorte de totémisme actif, répandu partout, créateur de Symboles et qui donne issue à toutes sorte d'applications. (8: 334)

\section{Rejoindre l'impossible (chora):}

Sa vie durant, Artaud a voulu sauter la barrière de la conscience (8: 277), et le Mexique sera l'élément détonateur qui lui permettra de détruire celle-ci. A cette époque (1936), il croit encore à l'anarchie sociale de l'art, comme il le déclare dans un article publié dans un journal mexicain:

L'art a pour devoir social de donner issue aux angoisses de son époque. L'artiste [...] est un bouc émissaire, [...] son devoir est d'aimanter [...] les colères errantes de l'époque pour la décharger de son mal-être psychologique [...]. (8: 287)

Mais, selon Artaud, l'Occident qui ne croit plus à l'art, dédaignant les valeurs spirituelles, est en train de se détruire. C'est pourquoi, lors de son voyage au Mexique, il est "en quête d'hommes politiques, non d'artistes," car ces derniers sont devenus des esclaves (8: 257). 
Bien que sympathiques à cette nouvelle idée de l'homme, les hauts fonctionnaires mexicains regardaient d'un oeil différent l'avenir de leur pays: une conjonction des intérêts de la haute bourgeoisie et des Etats-Unis, ainsi qu'une dispersion des forces de gauche, fera avorter la situation prerévolutionnaire d'alors. Aussi l'insistance d'Artaud à parler des Indiens, à prôner l'adoption par le corps mexicain de la conscience indienne, fait-elle scandale dans le milieu blanc et métis. Déchu, il partira "à la recherche de l'impossible" à l'intérieur du pays (8: 361).

Après un long et pénible voyage, il arrive chez les Tarahumaras lesquels pratiquent le culte du peyotl qui correspond à un rite d'identification totale à la race, de rentrée en soi-même, conception séduisante pour Artaud. Quoique le gouvernement défende à ceux-ci leur pratique religieuse traditionnelle, il réussit à une cérémonie initiatique. Chez ces Indiens, Artaud découvre actualisées ses préoccupations personnelles. Ceux-ci, en effet, "rendent un culte à un principe transcendant de la Nature, lequel est Mâle et Femelle comme il se doit" (9: 83), comme la racine du peyotl qui est hermaphrodite, et ils "vivent comme s'ils étaient déjà morts" (9:97) dans le plus complet mépris de la réalité. Leur danse est cosmogonique, leur chant "un appel secret de je ne sais quelles forces obscures, de quelles présences de l'au-delà" (9: 93) et ce qu'ils redoutent le plus est la perte de leur double spirituel $(9: 86)$.

En plus des parallèles entre l'expérience peyotlique et celles des drogues qu'ingurgita Artaud pendant toute sa vie, celui-ci s'est probablement identifié à ce peuple, tout en projetant ses obsessions sur leur rituel. Quoi qu'il en soit, de retour en France, au coeur de l'Occident méprisable, après avoir trouvé, croyait-il, une culture où l'homme "inventait la vie", il se retrouve dans la misère et doit subir une cure de désintoxication que l'usage de l'héroïne avait rendue nécessaire. Une dernière déception amoureuse avec une jeune Belge et une conférence mal accueillie dans le pays de celle-ci amèneront Artaud à enfin entreprendre l'ultime expérience de la folie. Mais la tragique aliénation d'Artaud dans cinq asiles entre 1937 et 1946, ses déboires aux mains des psychiatres et sa rèhabilitation partielle vers la fin de sa vie, ne nous intéressent que par leurs "traces" dans l'oeuvre, extériorisation hors de tout contexte d'un texte sémiotique. 
Rejeté entièrement de la société, castration externalisée dans l'électrochoc "afin de le mettre sous le coup de la loi" (12: 212), Artaud va refuser à son tour toute attache sociale: avec lui, "ni tutoiement, ni copinage jamais, pas plus dans la vie que dans la pensée" (12: 207). Il rejette la famille ("L'Exécration du père-mère") et il écrit sa propre épitaphe (Ci-gît), car pour lui, l'enterrement correspond à un assassinat. Antonin Artaud "n'est plus" (10:59), écrit-il, il est mort à l'asile de Ville-Evrard au mois d'août 1939 (10: 71), Antonin Nalpas a pris sa place; plus tard, il sera remplacé par Artaud le Mômo.

Avant son voyage "mystique" en Irlande, où il "perdra la raison", déjà il avait publié D'un voyage au pays des Tarahumaras, sans nom d'auteur, et signé du surnom "Le Révélé" Les Nouvelles révélations de l'être, écrit à partir de deux lectures de Tarot, dans lequel il avouait avoir souffert jusqu'ici parce qu'il avait refusé de se jeter dans le Vide, de "quitte[r] la réalitê" (7: 149-51). C'est à l'asile de Rodez qu'il fait ce saut. Là, il est séparé non seulement du monde social, mais de son corps même. Un double intérieur va prendre sa place, un double pur, sans organes (13: 104), mais qui n'est pas encore parfait car non délivré de la malédiction du sexe, dernier lien avec l'extériorité. Cette dernière lutte contre la sexualité est reliée à l'ambivalence d'Artaud vis-à-vis de la religion catholique:

Sans la sexualité, dit le Mal, il n'y aurait jamais eu d'enfants et la Race humaine ne pourrait plus croitre." Cela veut dire que la Race humaine a un jour préféré de croître en passant par les égouts au lieu de franchir l'ineffable pont d'arches au delà duquel l'IMMACULE INFINI invoque l'ETERNITE VIERGE qui ne cesse de le contempler. (10: 122)

D'après le docteur Ferrière, l'attitude d'Artaud varie: l'aumônier de Rodez "ne savait jamais s'il verrait le poète recueilli à tous ses offices ou s'il serait injurié par lui [...] (Brau 212). Cette dichotomie entre le pur/impur, d'où relève le religieux/irréligieux, est à la base de l'identité sexuelle. L'enfant alors partagé entre le désir pour la mère et la négation de son désir par le langage (le Nom/Non du Père) apprend à sacrifier l'appel de son corps au profit de la symbolisation 
et de la sublimation. L'infatuation religieuse d'Artaud est le prolongement de la subordination de la puissance d'attraction maternelle sous l'ordre patriarcal logique et symbolique.

Il faut aussi voir dans son attirance pour le christianisme, le dernier lien à la fois l'éloignant et l'approchant de la chora. Le sacré, en l'assujettissant à un système où l'UN règne, le protège contre l'ultime saut dans la folie sémiotique, mais en même temps le rapproche d'elle, car le péché, souillure chrétienne, provient d'une réconciliation partielle avec le principe maternel oral ( $c f$. l'eucharistie). L'impur, l'abjection, qui agissait de l'extérieur dans la religion juive, s'est intériorisé dans le christianisme, dẻsir et péché, sexe et religion s'amalgamant: "[...] érotique et sombre, [...] toute messe dite est un acte sexuel de plus dans la nature dégagée." (13: 258)

Signalons que, pour Artaud, la célébration de la messe avait sûrement alors un pouvoir de fascination car elle comportait des rites cathartiques et pouvait ainsi se rapprocher du "thêatre de la cruauté." Les rites, selon Kristeva, illustrent la frontière, l'entre-deux spatial, où se rencontrent l'autorité sémiotique (du corps, par la mère) et la loi symbolique (des signifiants, par le père) (Pouvoirs, 88).

\section{Artaud "éclaté":}

Dans ce tiraillement, Artaud, nous l'avons vu précédemment, meurt maintes fois, pour enfin ressusciter "pur corps", sans esprit. Dans le "Dossier d'Artaud le Mômo", l'on trouve résumé ce triomphe du corps sémiotique sur le rationnel patriarcal et castrateur:

[...] Lintelligence est venue après la sottise, laquelle l'a toujours enculée de près, [...] l'esprit est ce qui passe son temps à savoir ce que le cul peut faire / et pour le mener et pour lui prendre ses trucs [symbolisation-castration], / d'ailleurs ce n'est pas un esprit qui a trouvé les choses mais un corps qui pour être avait besoin d'exister / et ce corps s'appelait moi, / moimême, / le désintéressé de tout intérêt, [...] 
Le moi est ce qui veut conserver son être / non dans l'absolu mais dans le particulier, / ennemi-né de l'éternité [Dieu, Religion], / que le particulier se renouvelle non à l'infini / mais dans l'indéfini indéfiniment multiplié [la choral, / c'est un ètat peau chair / que peut l'interne utérin du sexe femme, le moi, [...]

Ne pas entendre car la discussion c'est de l'esprit, / la définition de la situation envisagée, / c'est le père et le fils / poutrer, crotter, / les esprits n'en peuvent mais. / Et maintenant un peu d'orgueil [renaissance sémiotique] / (délire, hallucination, mégalomanie, etc., etc.).

Je suis, moi, l'immortalitè, l'innéité, la pérennité, / et aussi la sublimité. / Est-ce une tare? Alors, dans ce cas je suis taré. / Car qu'est-ce que c'est que la sublimité? / L'outrance du subliminal outragé, / à force d'avoir été outré / pour avoir voulu être outrepassé, / par tout ce qui était limitatif [...]. (12: 181-85)

[...] vous verrez mon corps actuel voler en éclats / et se ramasser sous dix milles aspects / notoires / un corps neuf / où vous ne pourrez / plus jamais / m'oublier. (13: 118)

Cet Artaud "renouvelê", sans antécédent ("je suis mon fils, mon père, ma mère / et moi" [12: 259]), dont l'esprit sera maintenant à la dérive du corps, coïncide presque avec sa sortie de l'asile, en 1946, comme si cette dernière "évolution" devait se réaliser en toute liberté.

Lui qui avait tant de difficulté à écrire est alors pris d'une boulimie d'écriture. Il commence par renier ce qu'il a écrit "dans l'état mental stupide du converti que les envoûtements de la prêtraille profitant de sa faiblesse momentanée maintenaient en état de servitude" (9: 39). Maintenant, 11 "choisilt les choses en fonction de [lui] 
/ et non avec le mental / mais avec la bedaine ventrière de la fureur" (12: 200). Il se voit "contraint / de dire non, / NON / alors / à la négation [...primaire-secondaire qui avait] pressé / jusqu'à la suffocation / en [lui] / de l'idée de corps / et d'être un corps" (13: 96-97), et c'est alors la tombée dans un souterrain féminin d'où il ne sortira plus; réalisant ainsi un vieux désir de n'être "Plus jamais dans le Masculin" (4: 179).

L'oeuvre d'Artaud, à partir de cette période, subit une transformation radicale: écriture cathartique, organique: “[J]e veux n'écrire que quand je n'ai plus rien à penser. Comme quelqu'un qui mangerait son ventre, les vents de son ventre par dedans" (cité dans Brau, 228). L'on retrouve ici les deux composantes de la réaction de l'enfant frustré lors du refoulement primaire: une agressivité sadique, jouissance de la destruction et pulsion de mort, et, par le rejet du langage et de la symbolisation, une réactivation de l'analité désenfouie.

Ne voulant s'identifier à aucun parent, sinon à lui-même, il s'auto-érotise dans la paranoia obscène la plus destructive, particulièrement dans Pour en finir avec le jugement de Dieu. Lacte d'écrire devient jouissance à tout démolir, à rejeter et à expulser des excréments. Que le symbolisme (et le langage) soit de l'anal, Artaud le savait:

[...] le vieux [...] n'a pas voulu lâcher / le principe

/ du verbe / qu'il tenait sous sa langue / VISSEE, / verte, / viride, / virtée, viridée, / vendue. / Son idée a êté donc de maintenir / le corps humain / sous la dictature de cette coprophagie funèbre, / de cette funéraire déglutition dégustatoire d'excréments [...]. (13: 224)

Ce passage sur la poésie et la religion est encore plus significatif:

Le sexe est sombre,[...] la poésie l'est encore plus. [...] Ce siècle ne comprend plus la poésie fécale, l'intestine malheur, de celle, Madame Morte, qui depuis le siècle des siècles sonde sa 
colonne de morte, sa colonne anale de morte, dans l'excrément d'une survie abolie, cadavre aussi de ses moi abolis, et qui pour le crime de n'avoir pu être un être, a dû tomber pour se sonder mieux être, dans ce gouffre de la matière immonde et d'ailleurs si gentiment immonde où le cadavre de Madame Morte, de madame utérine fécale, madame anus, géhenne d'excrément par géhenne, dans l'opium de son excrément, fomente fama, le destin fécal de son âme, dans l'utérus de son propre foyer. L'âme, dit le corps enterré de l'être, tombe, fécal comme un excrément et s'entasse dans son excrément. J'ai vu devant moi de beaucoup de cercueils tomber je ne sais quelle matière noire, quelle immortelle urine de ces muets de vivre, qui miettes de matière, en miettes, goutte par goutte, s'abolissaient. Le nom de cette matière est caca, et caca est la matière de l'âme, dontj'ai vu tellement de cercueils répandre leurs flaques devant moi. Le souffle des ossements a un centre et ce centre est le gouffre Kah-Kah, Kah le souffle corporel de la merde, qui est l'opium d'éternelle survie. [...] Vivre c'est éternellement se survivre en remâchant son moi d'excrément, sans nulle peur de son âme fécale, force affamante d'enterrement. [...] (9: 191-93)

Nous assistons à la rupture du phéno-texte linéaire, laissant passer le géno-texte (impulsion verticale) dans le signifié, exactement comme la culture des Tarahumaras dans laquelle Artaud a reconnu le même principe de vie:

c'est le seul endroit de la terre, qui nous propose une vie occulte, et la propose à la surface de la vie. (8: 206) 
[A propos du rite du peyotl:] Car cette avance dans la maladie [et le mal] est un voyage, une descente pour RESSORTIR AU JOUR. (9: 56)

Si le retour de l'analité non sublimée transforme le contenu du signifié, sous cette pulsion, la linéarité signifiante subira une altération encore plus radicale: déformations de mots, répétitions, hyperkinésie, stéréotypie, interjections... Artaud joue avec la graphie et le rythme des phrases augmente d'importance, leur oralité s'amplifiant jusqu'à l'éclatement complet de toute signification laissant apparaître une nouvelle langue, la sienne:

Tout vrai langage / est incompréhensible [...]. (12: 95)

Exclamations, / interjections, cris, / interruptions, interrogations / sur / la remise en cause / du / Jugement Dernier. [...]

La peur de vivre. / koerman / ta / radaborsta / taborsta / radaborsta / santa pan / Nous ne savons rien de la vie, nous. (13: 233-34)

Fu fe tou fais, outo, quand tou fais ca, / tu enlèves les zesprits d'oun d'air, / alors t'es pas ancore gari, tou crois encou aux zesprits.

Jou vous dit que la vie est malade, / la vie est très malade. (13: 249)

van Gogh / van Gogh / mouen dabi / taouen arbi saled / mawen dibi / tauten libifa

En dehors de cela tous les hommes de cette humanité ne sont que de la sale teigne d'emmerdeurs. (13: 227) 
Dans cette pulvérisation langagière d'un matériau aliénant, Artaud retrouve le "rythme" inconscient, pulsionnel; il introduit un élément ludique (enfantin), l'afflux de la jouissance dans l'ordre du langage. Il semblerait même que l'on ait à faire à de la musique et non à du délire, car Artaud était au courant des recherches phonétiques et son langage déconceptualisé était attribuable à de longues heures de travail et de correction. Il ne s'agit pas d'une impuissance de la part d'Artaud, mais d'un refus délibéré du premier système sur lequel tous les autres découlent, la langue.

Il faut voir de la même manière sa dernière apparition publique à cette maintenant célèbre conférence du Vieux-Colombier. Décharné devant une liasse de papier sur une table, les mains voltigeant, tordues, il dit ses "poèmes", parle de ses aventures en Irlande, des Tarahumaras. Soudain, le ton change, Artaud dénonce les forces du mal, la psychiatrie, l'électrochoc. Avec des cris, des éructations magnifiques, c'est l'envol des feuillets préparés, inutiles et aliénants. Ce délire verbal choqua à peu près tout le monde: ils n'avaient pas encore compris. Déjà l'homme Artaud s'était éclipsé derrière la légende. "L'homme qui a révolutionné Paris, Antonin Artaud, est le fils d'une femme et d'un démon," titre un journal. Il n'avait plus qu'à mourir dans la douleur comme il avait vécu, et dans l'anonymat. Les hommes de lettres étaient prêts avec leur "arsenal littéraire" à l'expliquer, à le "systématiser", comme nous l'avons fait, espérons du moins avec respect.

\section{Conclusion:}

Sans doute pourrait-on résumer les problèmes et les expériences singulières d'Artaud sous un vocable médical: une schizophrénie de plus en plus avancée scindant corps et intelligence. D'autres l'ont qualifié de mystique séculier, de rebelle visionnaire ou de disciple de la "pure conscience". Il ne nous revient pas d'en être le juge. S'il abandonne la littérature, au début de sa carrière, au profit du théâtre et du cinéma, c'est qu'il est convaincu de l'impuissance du langage à manifester la pensée. Il a voulu convaincre le public que l'esprit et la logique sont incompatibles, que la relation que le premier établit entre le monde des objets et lui-même n'est pas rationnelle, mais incontrôlable dans la mesure où la matière exerce une force sur la 
psyché. Peut-être retrouve-t-on là une conception animiste du monde, mais, à la suite des découvertes psychanalytiques et des interrogations postmodernes, il semble bien que la société, par le langage et tous les systèmes qui en dérivent, façonne l'esprit de l'homme et son comportement, et en fasse sa "machine-esclave".

La folie d'Artaud fut de croire qu'il pouvait seul sauver le monde de cette maladie de "rationalitè", maladie de forger les intelligences pour contrôler le feu de corps. Il chercha les points d'acupuncture pour insérer les aiguilles thérapeutiques. Le surréalisme lui offrait une voie qu'il déclina de suivre après son abâtardissement au marxisme. Avec son "théâtre de la cruautê" qui, selon plusieurs, fut la plus importante innovation théorique du théâtre au XXième siècle, il voulait retrouver la pureté théâtrale cathartique de l'antiquité, et même reproduire des rituels d'initiation ayant une valeur psychanalytique sociale. En dernier lieu, d'une façon tragique peutêtre, mais avec combien d'intuition, ses "oeuvres de folie" confirment ce qu'il avait affirmé plus jeune sur le rôle castrateur du langage et que Lacan découvrira par après.

A part peut-être les minces fascicules d'Héliogabale et de Van Gogh le suicidé de la société, l'oeuvre littéraire d'Artaud est inexistante. Mais c'est précisément ce manque qui en fait la véritable valeur, car en s'analysant sans répit Artaud nous a révélés à nous-mêmes. Dans ce "manque" inscrit au plus profond de la conscience, il a découvert son origine et, humblement, il a tenté d'atténuer ce néant dans un dynamisme révélateur:

Comme la vie, comme la nature, la pensée va du dedans au dehors avant d'aller du dehors au dedans. Je commence à penser dans le vide et du vide je vais vers le plein; et lorsque j'ai atteint le plein je peux retomber dans le vide. Je vais de l'abstrait au concret et non du concret vers l'abstrait.

Arrêter la pensée dehors et l'êtudier dans ce qu'elle peut faire c'est méconnaître la nature interne et dynamique de la pensée. C'est ne pas vouloir sentir la pensée dans le mouvement de 
son destin interne qu'aucune expérience ne peut capter.

J'appelle poésie aujourd'hui connaissance de ce destin interne et dynamique de la pensée.

Pour retrouver sa nature profonde, pour se sentir vivre dans sa pensée, la vie repousse l'esprit d'analyse où l'Europe s'est égarée. (8: 192)

Après tant de cris et de requêtes, aurait-il une fois de plus raison, comme les recherches de Roland Barthes et de Michel Foucault semblent l'indiquer? Il faut cependant être prudent si l'on poursuit cette voie sous peine d'être engouffré dans la douleureuse "déchirure" de l'abjection. Car si pour Artaud, "c'est la fin qui est le commencement" (12: 84), l'inverse fut aussi vrai: en régressant à ce "commencement" primordial, il trouva sa "fin". Paradoxalement, dans une "pure absence" de représentation, Artaud a cherché à "signifier", en dehors du système patriarcal, une "pure présence", retour au "sein maternel", au "paradis perdu" dont l'homme se souvient dans toute ses mythologies.

\section{Notes}

${ }^{1}$ D'après le docteur Ferrière, l'attitude d'Artaud varie: l'aumônier de Rodez "ne savait jamais s'il verrait le poète recueilli à tous ses offices ou s'il serait injurié par lui [...] (Brau 212)... Pour une analyse en détails de toutes ces notions psychanalytiques, voir Kristeva, "Le Sujet en procès" 43-70.

${ }^{2}$ Pour une définition plus complète de la chora en relation avec le sémiotique, voir Kristeva, Au commencement était l'amour 13-16, Pouvoirs de l'horreur 9-39 et "Le Sujet en procès" 45-46.

${ }^{3}$ Tout au long de la présente étude, le "trait oblique" (/) est utilisè pour indiquer les alinéas ou coupures du texte d'Artaud, procédé que le MLA Handbook suggère d'employer avec la poésie. 
${ }^{4}$ Cette dernière lettre fut largement diffusée en tract lors des événements de mai 1968.

${ }^{5}$ Jacques Henric, dans "Artaud travaillé par la Chine," développe ce côté artaldien d'une stratégie globale contre l'impérialisme. Relire aussi Les Messages révolutionnaires d'Artaud (Vol. 7).

${ }^{6} \mathrm{Ce}$ cancer à l'anus ferait parti du mythe Artaud, bien que les violentes douleurs intestinales, l'impossibilité de digérer ou de retenir la nourriture amènent à un diagnostic très près de la légende, son goût de l'analogie le poursuivant jusque dans la mort.

\section{Bibliographie}

Adamov, Arthur. "Introduction à Antonin Artaud." Obliques 10, 11 (1976): 5-7.

Artaud, Antonin, Oeuvres complètes. 24 vols. Paris: Gallimard, 1956-1988.

André-Carraz, Danièle. L'Expérience intérieure d'Antonin Artaud. Paris: Saint-Germain-des-Prés, 1973.

Brau, Jean-Louis. Antonin Artaud. Paris: La Table Ronde, 1971.

Camus, Michel. "Paul les Oiseaux ou la dramaturgie intime d'Artaud." Obliques 10, 11 (1976): 21-34.

Derrida, Jacques. L'Ecriture et la différence. Paris: Seuil, 1967.

Durozoi, Gérard. Artaud; l'aliénation et la folie. Paris: Larousse Université, 1972.

Foucault, Michel. Histoire de la folie à l'âge classique. Paris: Gallimard, 1972. 
Hahn, Otto. Portrait d'Antonin Artaud. Paris: Le Soleil Noir, 1968.

Kristeva, Julia. Au commencement était l'amour. Paris: Hachette, 1985.

. Pouwoirs de l'horreur. Paris: Seuil, 1980.

. "Le Sujet en procès." Sollers, Artaud. 43-133.

. Recherches pour une sémanalyse. Paris: Seuil, 1969.

Le Galliot, Jean. Psychanalyse et langages littéraires Paris: Nathan, 1977.

Heimonet, Jean-Michel. "Pour ne pas en finir..." Obliques 10, 11 (1976): 95-99.

Henric, Jacques. "Artaud travaillé par la Chine." Sollers, Artaud. 215-44.

Morel, Jean-Paul. "Artaud et le cinéma." Obliques 10, 11 (1976): 117-32.

Prieur, Jérome. "La Machine à l'oeil buté." Obliques 10, 11 (1976): 107-11.

Sollers, Philippe, ed. Artaud. Paris: Union générale d'éditions (Collection 10/18), 1973. 